"Die Wahrheit ist den Kindern zumutbar" Schuld und Bekenntnis

in Gudrun Pausewangs geschichtserzählendem Kinderund Jugendbuch

Ich war dabei

\title{
Geschichten gegen das Vergessen
}

\begin{abstract}
Was Literatur ist, darüber gibt es verschiedene Ansichten, doch eines stellt sie gewiß dar: das kollektive Gedächtnis der Menschen. Sie ist der Speicher, die umfassendste Sammlung von Erlebtem und Gedachtem, sie ist ein einzigartiger Vorrat an Welterfahrung.
\end{abstract}

LENZ 1992: 7

„Bald wird es keine Zeitzeugen mehr geben. Ich habe diese Geschichten geschrieben, um mit dazu beizutragen, dass die Verbrechen jener Zeit nicht in Vergessenheit geraten. Ein so unmenschliches Regime darf nie wieder Fuß fassen können - weder bei uns noch anderswo“", schreibt die deutsche Schriftstellerin Gudrun Pausewang (mit bürgerlichem Namen Gudrun Wilcke) im Nachwort ihres Buches Ich war dabei. Geschichten gegen das Vergessen (2004) und bringt damit zum Ausdruck, dass die in diesem Buch enthaltenen Täter- und Opfergeschichten aus der NS-Zeit Kindern zumutbar sind, oder wie Ingeborg Bachmann 1959 in Bonn anlässlich der Entgegennahme des „Hörspielpreises der Kriegsblinden“ versicherte: „Die Wahrheit ist dem Menschen zumutbar“. Die

\footnotetext{
1 Pausewang (2004: 154).
} 
Wahrheit, die weiterhin leben soll, und die Frage der Schuld in der Familie, die in diesen Geschichten immer wieder mitschwingt, sind in der deutschsprachigen Literatur nach wie vor aktuell und im kollektiven Gedächtnis verankert. Ebenso aktuell ist auch das Thema Bekenntnis bzw. Eingeständnis der persönlichen oder kollektiven Schuld, in dem Aleida Assmann eine Identitätswende sieht:

Das Bekenntnis zu nationaler Schuld bedeutet gerade nicht, wie viele befürchten, eine Befleckung des kollektiven Selbstbildes, sondern schafft die Möglichkeit einer Identitätswende, indem sich eine Nation ausdrücklich von den Verbrechen der eigenen Geschichte distanziert und zu zivilgesellschaftlichen Werten bekennt. ${ }^{2}$

Darauf verweist etwa eine beträchtliche Anzahl von literarischen Neuerscheinungen, die der Gattung des Familien- oder Generationenromans zugeordnet werden, einer Gattung, welche die Vergangenheit vom Ersten Weltkrieg bis zur heutigen Gegenwart umspannt und historischgesellschaftlich-familiäre Kontexte, verwoben mit einer Suche nach der Wahrheit und der eigenen Identität, in den Blick nimmt. ${ }^{3}$ Das Ergebnis von Pausewangs Auseinandersetzung mit der Wahrheit sind eigens für Kinder und Jugendliche verfasste Bücher, die einen bestimmten Abschnitt des 20. Jahrhunderts beleuchten und den Nationalsozialismus sowie die Schrecken des Zweiten Weltkrieges thematisieren. Diesbezüglich stellt sich die Frage, auf welche Art und Weise und mit welcher Absicht diese Themenkomplexe in die deutschsprachige Kinder- und Jugendliteratur von Pausewang aufgenommen werden? Bevor aber tiefer darauf eingegangen wird, seien noch einige Bemerkungen zur Gattungsbezeichnung und Klassifizierung der Kinder- und Jugendbücher gemacht und daran erinnert, wie und wann der Prozess der literarischen Geschichtsaufarbeitung für die Jüngsten in Gang kam. Der Blick soll sich dabei vor allem auf Pausewangs Euvre richten.

Nach den bisher entwickelten Definitionen lassen sich Pausewangs Kinder- und Jugendtexte ganz klar unter den Begriff „originäre Kinderund Jugendliteratur"4 (auch „spezifische Kinder- und Jugendliteratur") bringen. Von originärer Kinder- und Jugendliteratur wird laut HansHeino Ewers dann gesprochen, wenn literarische Texte seitens ihrer Verfasser von vornherein als Kinder- und Jugendlektüre bestimmt waren. Eine solche Festlegung wirkt sich auf die Textgestaltung aus, die als kind- und jugendgemäß (kind- und jugendgemäße sprachliche Ebene,

\footnotetext{
2 Assmann (2011).

3 JELEČ (2016: 147).

${ }^{4}$ Ewers (2012b: 19).
} 
entsprechende Form und Struktur, Gattungswahl, Stoffwahl, inhaltliche und motivische Ebene usw. ${ }^{5}$ ) zu bezeichnen ist. Kinder- und Jugendliteraturwissenschaftler Ewers hat in seinen Untersuchungen darauf hingewiesen, dass es sich bei der Kinder- und Jugendliteratur nicht um einen klar umgrenzten Bereich handelt und es demnach eine Mehrzahl von Bereichen gibt. ${ }^{6}$ Die geschichtserzählenden Kinder- und Jugendbücher, wie sie auch von Pausewang geschrieben werden, ist nur ein Bereich bzw. eine Möglichkeit, derartige Kinder- und Jugendbücher zu klassifizieren, welche dann auch aufgrund des Unterlaufens der Grenzen von Fiktion und Dokumentation das Beiwort „hybrid“ bekommt. ${ }^{7}$ Fakten und Fiktionen stehen in diesen Texten nebeneinander. In Bezug auf diesen Aspekt hat sich eine in der Kinder- und Jugendliteraturforschung allgemein akzeptierte Definition von Malte Dahrendorf durchgesetzt. Nach dieser Definition wäre sie als

diejenige - vor allem erzählende - Kinder- und Jugendliteratur [zu verstehen], deren Geschichten entweder vor konkretem zeitgeschichtlichen Hintergrund spielen, aber so, daß das „Gespielte“ ohne diesen Hintergrund so nicht möglich wäre [...], oder Zeitgeschichte ausdrücklich thematisieren, so daß Figuren und Handlungen mehr oder weniger zum Vorwand werden können. Mit „Zeitgeschichte“ ist die jüngste oder jüngere Vergangenheit gemeint, eine Vergangenheit, in deren unmittelbarer Auswirkung wir heute noch leben, zum Beispiel: Drittes Reich und seine Vorgeschichte, Zweiter Weltkrieg, Ost-West-Spannung, Konflikte der Dekolonialisierung und der Dritten Welt, Rassismus [...]. ${ }^{8}$

Das für diesen Beitrag ausgewählte Buch Ich war dabei. Geschichten gegen das Vergessen ist ein weiteres Beispiel der originären Kinder- und Jugendliteratur mit konkretem zeitgeschichtlichem Hintergrund. Die Abbildung von Geschichte für kindliche und jugendliche Empfänger, die Darstellung von Ereignissen rund um den Zweiten Weltkrieg sowie die Vergangenheitsaufarbeitung auf jugendliterarischer Ebene rechtfertigen diese Einordnung. Allerdings war das nicht immer so: Die ersten Nachkriegsjahre waren gekennzeichnet durch eine fehlende Auseinandersetzung mit der NS-Vergangenheit. Vor allem die 1950er Jahre gelten als eine Zeit des Schweigens über die nationalsozialistische Vergangenheit. In Deutschland gab es in diesen Jahren keine Kinder- und Jugendbücher, die diese Zeit literarisch verarbeiteten. Die nationalsozialistische Vergangenheit wollte erstmal nicht erinnert werden und das hatte folglich auch

\footnotetext{
${ }^{5}$ EWERs (2012b: 175-195).

${ }^{6}$ EWERs (2012a).

7 AssmanN (2006: 27).

8 DAHRENDORF (1997: 205).
} 
Auswirkungen auf deutsche kinder- und jugendliterarische Werke, wo es zunächst kaum Abweichungen von diesem Trend gab. Aden-Grossmann konstatiert hierauf Bezug nehmend:

Ganz überwiegend war die KJL der Nachkriegszeit zwischen 1945 und 1959 apolitisch und folgte damit dem allgemeinen gesellschaftlichen und literarischen Trend, diese Epoche zu verdrängen: Es wurden überwiegend harmlose Geschichten publiziert, in denen eine „heile Welt“ dargestellt wurde. ${ }^{9}$

Erst mit größerem zeitlichem Abstand also finden die Erfahrungen des Zweiten Weltkrieges Eingang in die Kinder- und Jugendliteratur. „Rückwärtsgewandt“, „verdrängend“ oder „heile Welt“ sind Stichwörter, die diese Tendenz in der deutschsprachigen Literatur, die noch viele Jahre nach dem Zweiten Weltkrieg fortdauerte, am treffendsten beschreiben. So war man auch in der Kinder- und Jugendliteratur der Überzeugung, man müsse das junge Publikum ,vor dem zersetzenden Intellekt" ${ }^{\text {“10 }}$ behüten.

Die Publikationszahlen an Kinder- und Jugendbüchern zum Thema Nationalsozialismus und Holocaust begannen im deutschsprachigen Raum erst in den 1960er Jahren allmählich zu steigen, um in den darauffolgenden Jahrzehnten immer mehr zuzunehmen. Die „neue“ Kinder- und Jugendliteratur setzte sich nun zum Ziel, die Vergangenheit der deutschen Geschichte, welche lange Zeit verdrängt wurde, zu enttabuisieren bzw. das kollektive Wissen zu bewahren und zu tradieren, um so letztendlich auch die Bewusstmachung und Überwindung kollektiver Schuld möglich zu machen. Richtungweisend war sicher Karl Bruckners erfolgreichstes Buch Sadako will leben (1961), das Weltgeschichte anhand eines Einzelschicksals erzählt, von wehrlosen Kindern, die die Kriegsgeschehnisse in Japan zur Zeit des Zweiten Weltkrieges erleben. Im selben Jahr erschien Hans Peter Richters Jugendbuch Damals war es Friedrich, eines der ersten Jugendbücher zum Thema Judenverfolgung. Nennenswert ist ferner Winfried Bruckners Roman Die toten Engel (1963), der 1940 spielt und das Schicksal der Juden thematisiert, oder etwa der Roman Das Schattennetz (1964, die zweite Fassung erschien 1981 unter dem Titel Geh heim und vergiss alles) von Käthe Recheis, der autobiographische Züge trägt. Nach Angaben Rüdiger Steinleins ${ }^{11}$ wurden im gesamten deutschsprachigen Raum bis zum Jahr 1996 über 1000 Titel veröffentlicht, die Themen wie Krieg, Verfolgung, Flucht, Antisemitismus, Täter- und Opferschaft u. Ä. behandeln.

\footnotetext{
9 Aden-Grossmann (2000: 285).

10 WILD (2002: 302).

11 STEINLEIN (1996: 87).
} 
Wirft man einen Blick auf Pausewangs Publikationen, insbesondere im Bereich der Kinder- und Jugendliteratur, dann fällt auf, dass sie diese Themen viel später zu problematisieren beginnt. Erst in den späten 70er Jahren behandelt sie Themen wie Krieg, Täterschaft, Schuld, NS-Alltag, Flucht und Verfolgung, Kampf ums Überleben, Emigration, Holocaust und Neonazismus und veröffentlicht vor den Geschichten gegen das Vergessen folgende Prosawerke für Kinder und Jugendliche aus dem genannten Themenfeld: Auf einem langen Weg (1978), der als Vorlage für eine Filmproduktion diente, schildert die Geschichte von zwei Brüdern, die in den letzten Monaten des Zweiten Weltkrieges von der Mutter getrennt werden und sich durch das vom Krieg verwüstete Deutschland bis in den Westen zu ihrer Tante durchschlagen. Ihre eigenen Erfahrungen mit Krieg und Vertreibung verarbeitete Pausewang in der autobiographischen Trilogie Rosinkawiese (1980), Fern von der Rosinkawiese (1989) und Geliebte Rosinkawiese (1990), zudem tragen Werke wie Wiedersehen mit Anna (1997) autobiographische Züge. Der Roman Reise im August (1992) schildert die Deportation eines jüdischen Mädchens in das Konzentrationslager Auschwitz, Der Schlund (1993) und Die Meute (2006) die Gefahren einer weiteren rechtsnationalen Diktatur. Im Jahr 1997 schreibt Gudrun Pausewang ein Buch über die Jugendjahre Adolf Hitlers Adi-Jugend eines Diktators und auch die darauffolgenden Werke $D u$ darfst nicht schreien (2000), Macht euch euren Krieg allein! - und andere absichtliche Geschichten (2000), Überleben! (2005) und Der einhändige Briefträger oder ein Herbst, ein Winter, ein Frühling (2015) kreisen thematisch um den Zweiten Weltkrieg. In den Jahrzehnten davor schreibt sie überwiegend Bücher mit Handlungsorten in fernen Ländern, wie Chile oder Bolivien, von denen die Autorin fasziniert war. Einerseits kann dies mit ihren langjährigen Aufenthalten und ihrer Berufstätigkeit in verschiedenen Ländern Südamerikas und dem daraus hervorgehenden Interesse für das südamerikanische Leben in Verbindung gebracht werden. Zweimal reiste sie nämlich nach Südamerika und lehrte an Schulen in Chile, Venezuela und Kolumbien; das erste Mal von 1956 bis 1963, das zweite Mal von 1967 bis 1972. In diesen zwölf Jahren bereiste sie den amerikanischen Doppelkontinent und sammelte Eindrücke und Erfahrungen, die sie später in ihren Büchern verarbeitete, so in ihrem ersten Roman Rio Amargo oder das Ende des Weges (1959), der an Erwachsene gerichtet war. Im Mittelpunkt ihres Interesses standen lange Zeit also gegenwärtige Probleme in Südamerika und Themen wie Umweltkatastrophen, Risiken der Nukleartechnologie (ökologische Kinder- und Jugendliteratur) und der Frieden ganz allgemein. Der Grundgedanke dabei war, das Lesepublikum zum Handeln aufzufordern bzw. die Bewusstmachung von Umweltproblemen und ih- 
ren möglichen Folgen für die Menschheit, als auch das Verstehen von ökologischen Zusammenhängen. Andererseits kommt aufgrund der im Vergleich zu den bereits genannten deutschen Jugendbuchautoren späten Vergangenheitsaufarbeitung in Pausewangs Kinder- und Jugendtexten die Frage auf, ob das Behandeln von Problemen der Dritten Welt, wie auch ihre Sehnsucht nach fernen Ländern, die sie schon in der Jugend entwickelte, und danach die späteren langjährigen Aufenthalte in Südamerika nicht als Flucht vor den eigenen Erlebnissen aus der NS-Zeit und der trostlosen Nachkriegsatmosphäre betrachtet werden könnten. Die Zeit in Südamerika könnte jedoch ebenso als ihre „Zeit des Umdenkens“ betrachtet werden, die ihr die nötige Distanz zu Deutschland gewährleistete, und diese wiederum eine andere Perspektive auf jene Deutschen, die nach dem Krieg in rechtfertigender Manier behaupteten, von nichts gewusst zu haben. ${ }^{12}$

\section{Gegen das eigene Vergessen}

Pausewangs eigene Kindheit und Jugend fällt in die im Buch thematisierte zeitgeschichtliche Epoche. Sie räumt offen ein, schon früh mit nationalsozialistischer Indoktrination in Berührung gekommen zu sein. Die 1928 geborene Autorin war bei Kriegsausbruch elf und bei Kriegsende siebzehn Jahre alt. In ihrer Kindheit leidet sie zunächst unter mangelndem Selbstbewusstsein, findet keinen Anschluss und die nötige Anerkennung. In dieser Zeit wird sie Mitglied einer Hitlerjugend-Organisation, die ihr zum ersten Mal das Gefühl von Zusammenhalt gibt und ihre Begeisterung weckt:

Ich war deprimiert. Ich hatte weiterhin keine Freunde, [...] ich habe Angst davor gehabt, zurückgewiesen zu werden. Und in der Klasse war ich Außenseiterin. Das einzige, was mir Spaß machte, das war aber auch wieder das Künstlerische, das war zum einen Lesen, Lesen, Lesen. Zum anderen war es die Mitgliedschaft in einer ganz besonderen HJ-Organisation und zwar nannte sich das „Sing- und Spielschar“, und war die einzige Gruppe, wo Jungen und Mädchen zusammen waren, und es waren nur die Vierzehn- bis Achtzehnjährigen. ${ }^{13}$

Im Nachwort macht Pausewang auf die biographische Prägung ihrer Geschichten aufmerksam und legt das auch mit der Wahl des Buchtitels Ich war dabei nahe. Manche der erzählten Geschichten gegen das Verges-

\footnotetext{
12 L'AвbаTe (2014: 14).

13 Pausewang (1999: 7).
} 
sen hat sie in ihrer Kindheit und Jugend selbst erlebt, was die Spuren im Narrativ erklärt. Sie erinnert sich dabei an ihren Vater, der in den Jahren vor dem Zweiten Weltkrieg und während des Zweiten Weltkrieges überzeugter Anhänger der Nationalsozialisten war. Er starb 1943 im Krieg, als die Autorin fünfzehn Jahre alt war. Als Mädchen glaubte sie selbst an die Ideen des Nationalsozialismus, sie wurde, wie sie selbst festhält, „zum kleinen Nazi erzogen“14. Sie erinnert sich zudem, am 30. April 1945, Hitlers Todestag, verzweifelt geweint zu haben. Sie lernt aus der Vergangenheit und erklärt in einem Interview: „Ich habe hinterher eingesehen, dass die Nazis unseren jugendlichen Idealismus missbraucht haben, und beschlossen, als Demokratin mitzuhelfen, dass es in Deutschland nie wieder zu solchen politischen Verhältnissen kommen wird“"15. Ihre Hilfe zeigt sich in der literarischen Auseinandersetzung mit der Machtübernahme der Nationalsozialisten, in der Darstellung von Tätern und Opfern, der Vermittlung von Geschichtsbewusstsein, geschichtlicher Belehrung und in der Bewusstmachung historischer Orte und Ereignisse. Gewiss beruhen die Geschichten nicht auf einem autobiographischen Pakt, dennoch ist die Nähe zur Autorin und ihren eigenen Kindheitserfahrungen vorhanden. Das belegt nicht nur das beigefügte Nachwort, sondern auch die Äußerungen der Autorin in zahlreichen Gesprächen zu diesem Thema. ${ }^{16}$ Für Pausewang, wie für viele andere Autoren, die während der NS-Zeit aufgewachsen sind und heute ihre Erfahrungen literarisch verarbeiten, erweisen sich das Schreiben und Erzählen aus der Kinderperspektive - mit mehr oder weniger autobiographischen Zügen - als eine typische Schreibweise des Erinnerns bzw. Methode der Geschichtsvermittlung, eine Form des Gedenkens und der Vergangenheitsbewältigung - man denke hier z.B. an den deutschen Lyriker, Kinder- und Jugendbuchautor Hans Baumann (1914-1988), den Autor des Liedes Es zittern die morschen Knochen, das 1932 während einer Wallfahrt nach Neukirchen bei Heiligenblut für die katholische deutsche Jugend entstand und von dem auch Pausewangs Kindheit bestimmt wurde. Im Nachhinein bekennt Baumann seine Schuld und setzt

14 ANONYM (2013).

15 RAMM (2015).

${ }^{16}$ Gleich am Anfang des Gesprächs mit Daniel Ramm zum Beispiel erklärt die Schriftstellerin, dass sie als Kind unter dem Einfluss des Nationalsozialismus stand, dass sie sowohl bei den Jungmädeln als auch beim Bund Deutscher Mädel war. Ferner erwähnt Pausewang im selben Gespräch, dass sich ihr Vater schon zu Beginn des Zweiten Weltkriegs als überzeugter Nazi freiwillig zum Wehrdienst meldete und fest an den Sieg glaubte. Diese Erfahrungen wirkten sich auf ihre Texte aus. Die Verführung durch den Nationalsozialismus hat die Autorin geprägt. Ihre Figuren sind ebenso davon betroffen. Vgl. RAMm (2015). 
sich 1961 in Der Bär und seine Brüder mit seinem Tun in der Zeit des Nationalsozialismus und seinem Gewissen auseinander, warnt vor Irrwegen und Führergestalten. ${ }^{17}$ Die biographische und literarische Erinnerung an die Kindheit im Nationalsozialismus läuft aber nicht nur auf eine Warnung für zukünftige Generationen hinaus, sondern bleibt, wie Hetzer feststellt, in der Suche nach einer Entlastung der Schuldgefühle, die sich von der Tätergeneration auf die Nachgeborenen überträgt, verhaftet. ${ }^{18}$ Natürlich wäre im Hinblick darauf die Frage zu stellen, ob die autobiographisch geprägten Geschichten der Autorin aus dem Jahr 2004 nicht eine verzögerte Vergangenheitsaufarbeitung auf jugendliterarischer Ebene darstellen. In einem Interview erklärt Pausewang:

Wir, die Autoren heutiger zeitgeschichtlicher Kinder- und Jugendliteratur, waren im Krieg selber noch Kinder. Als der Krieg vorbei war, standen wir rein biologisch auf der Schwelle zum Erwachsensein. Doch waren wir es schon als Kinder geworden, weil der Krieg uns nicht hat Kind sein lassen. Als er vorbei war, musste all das erst einmal verarbeitet werden. Nur mit einer entsprechenden Distanz lassen sich die Dinge, die Welt und das eigene Leben entsprechend reflektieren. ${ }^{19}$

\section{Geschichten über die Geschichte}

Gleich zu Beginn von Pausewangs Geschichtensammlung wird in die Vergangenheit geblickt. Es handelt sich dabei um eine Sammlung von insgesamt zwanzig Geschichten, die entweder aus der kindlichen, jugendlichen oder aus der Perspektive eines erwachsenen erinnernden Ichs dargestellt werden, in welchen die NS-Täterschaft, die Mitwisserschaft, die Ausgrenzung der Juden, die ideologische Verführung durch die NSPropaganda und die Mitgliedschaft bei den Jungmädchen behandelt werden. Das entspricht etwa Christine Nöstlingers Forderung in ihrer Rede anlässlich der Österreichischen Buchwochen im Jahr 1985: „Die Kinderund Jugendschriftsteller müssen mündiger werden, ihre Bravheit ablegen, ihre Kompromissbereitschaft auch. Sie müssen es lernen, auf der Hut zu sein, um nicht für Anliegen missbraucht zu werden, die nicht die ihren sind“20. Diese Aussage scheint ganz im Sinne Pausewangs zu sein. Neunzehn Jahre später setzt sie ihr eigenes Anliegen durch und schreibt ihre

\footnotetext{
17 WILD (2002: 310).

18 DINER (1987: 188-189).

19 KeLm (2006: 128).

20 NÖSTLINGER (1986/87: 158).
} 
Geschichten gegen das Vergessen entgegen allen Trends. Schon das Titelbild lässt das Thema vermuten. Zu sehen ist ein Appell-Platz, umrundet von gleich gekleideten, jungen Mädchen mit Zöpfen, die auf die Hakenkreuzfahne blicken und mit Begeisterung und Überzeugung den rechten Arm hochstrecken. In neun der zwanzig Geschichten spricht eine namenlose Ich-Erzählerin. Im Mittelpunkt ihrer Erzählungen stehen Einzel- oder Familienschicksale, wobei die Figuren mit der familiären Verstrickung in die Nazi-Welt, dem negativen Bild von Juden und dem antisemitischen Alltag konfrontiert werden. Oftmals zeigen sie sich verängstigt, verwirrt, unsicher und überfordert, was etwa an der Bemerkung „oft verstand ich nur die Hälfte oder noch weniger“ ${ }^{21}$ der Ich-Erzählerin in einer Geschichte erkennbar wird. Die Autorin zeigt darüber hinaus die Beeinflussbarkeit von Kindern und Jugendlichen. Die folgenden Abschnitte widmen sich der Analyse und Interpretation von sechs ausgewählten Geschichten.

In der Rahmenhandlung der ersten Geschichte, die den Titel Er war noch warm (7-12) trägt und mit sechs Seiten zu den kürzesten im Buch gehört, wendet sich eine in der Bahn sitzende alte Frau an die ihr gegenüber sitzende Ich-Erzählerin, die sich gerade Gedanken über Themen für ihr nächstes Buch macht und die Leser an die Autorin selbst denken lässt. Die alte Frau berichtet der namenlosen Ich-Erzählerin von ihren Erlebnissen aus der Kindheit und wird damit zur Erzählerin der Binnenhandlung. Ihre Erinnerungen reichen zurück bis ins Jahr 1941, als sie damals als ein 14-jähriges Mädchen beobachten musste, wie die letzten jüdischen Familien aus ihrem Wohnhaus geholt wurden. Der Titel der Geschichte bezieht sich auf den Eintopf in der Wohnung einer jüdischen Familie, die nicht mehr dazu kam, ihn zu essen, weil sie von „uniformierten Männern“22 abgeholt wurde. Am Rande sei bemerkt, dass die „uniformierten Männer“ zu den wenigen Ausdrücken im Buch gehören, die mit der NS-Zeit in Verbindung gebracht werden können. Die nichtjüdischen Nachbarn zeigen sich begeistert und stürmen in die Wohnung, um sich zu nehmen, was sie brauchen:

Als wir im halbdunklen Treppenhaus standen, sahen wir die Leute in die einzelnen Räume stürzen. Und schon kamen die ersten wieder zurück, schwer beladen: Frau Beck, unsere Nachbarin, hatte zwei Federbetten unter den Armen, Frau Möller von schräg gegenüber erschien auf einmal in Frau Birnbaums Pelzmantel, der alte Herr Lotterbach schleppte den verschnörkelten Lehnsessel, in dem der alte Herr Birnbaum immer gesessen hatte, zur Tür hinaus. ${ }^{23}$

\footnotetext{
21 Pausewang (2004: 69).

22 Pausewang (2004: 8).

${ }^{23}$ Pausewang (2004: 10-11).
} 
Die alte Frau erinnert sich, dass ihre Mutter am schnellsten war, sie hat dieses Ereignis nämlich schon ungeduldig erwartet, nahezu herbeigesehnt und war auch entsprechend darauf vorbereitet. Auch ihre Kinder mussten schnell handeln und sich schnellstmöglich mit der Mutter an den bereits gedeckten Esstisch setzen, um so die Wohnung zu besetzen:

Ein paar Mal ging die Küchentür auf, Gesichter schauten herein, grinsten, verschwanden wieder. [...] Als jemand die Tür aufriss, rief [die Mutter]: „Hier waren wir die Ersten!“ Die Tür ging wieder zu. [...] „Gesegnete Mahlzeit“, sagte sie, senkte ihren Löffel in den Eintopf und kostete. „Und er ist noch schön warm“, fügte sie zufrieden hinzu. ${ }^{24}$

Das älteste Kind in dieser Geschichte ist verwirrt, mit Verwunderung befolgt es die Anweisungen der Mutter. Der Bericht der alten Frau wird am Ende nicht kommentiert und es bleibt offen, ob die Autorin mit dieser Geschichte ein tatsächlich stattgefundenes Gespräch rekonstruiert.

Außerhalb des Elternhauses wurden Kinder und Jugendliche in Schulen und verschiedenen Freizeitorganisationen nach nationalsozialistischen Maßstäben erzogen. Die autobiographisch geprägte Geschichte Ein Novembermorgen (35-38) ist die fünfte Geschichte in der Sammlung und ein weiteres Beispiel dafür, welche Bilder den Schülern vermittelt wurden:

Die Juden, das hatten wir schon in der Schule gelernt, waren böse Menschen, die die braven Deutschen nur ausnutzen und übers Ohr hauen wollten. Wir hatten gelernt, dass man mit Juden nicht befreundet sein dürfe, weil sie nur lögen. Wir hatten auch gelernt, dass man mit ihnen kein Mitleid haben dürfe, weil sie es wegen ihres bösen Charakters nicht verdienten. ${ }^{25}$

Die ideologische Beeinflussung von Kindern ist auch das Thema der Geschichte Märchenstunde (45-50). Als die Ich-Erzählerin nach fünfundsechzig Jahren wieder vor ihrer ehemaligen Schule steht und sie von außen betrachtet, erinnert sie sich an eine Lehrerin, die gerne Märchen und andere Geschichten erzählte. Viele Jahre später erkennt die Ich-Erzählerin, dass die Lehrerin damals für die Verbreitung eines negativen Judenbildes, vor allem aber für die Verbreitung von Gedanken und Gefühlen der Angst unter den nichtjüdischen Schülern mitverantwortlich war. Sie macht mit ihrer verspäteten Feststellung deutlich, dass die Ausgrenzung der Juden während des Nationalsozialismus auch Lehrer zu verantworten hatten. Eine der zahlreichen Geschichten, die die Lehrerin damals in

\footnotetext{
24 Pausewang (2004: 10).

25 Pausewang (2004: 35).
} 
der Märchenstunde erzählt hat, handelt von einem Zahnarzt, der „eine krumme Nase, schwarze Locken, eine fleischige Unterlippe und große abstehende Ohren“"26 hat. Anhand der Beschreibung erkennen die Schüler schon während des Zuhörens, dass es sich um „kein[en] gewöhnlich[en] Mensch[en] “27 handelt, sondern um einen Juden, von dem sich zwei deutsche Mädchen, Ilse und Rosemarie, wegen Zahnschmerzen behandeln lassen möchten. Ilse wird in den Behandlungsraum gerufen. Kurze Zeit später hört Rosemarie im Warteraum ihre Schreie und im Anschluss nur Stille. Nun ist Rosemarie an der Reihe. Als der jüdische Zahnarzt sie holen möchte, gelingt es ihr noch zu fliehen. Die Frage einer Schülerin, was mit dem ersten Mädchen passiert sei, beantwortet die Lehrerin mit den Worten: „Macht euch Gedanken darüber...”"28. Aus den Erinnerungen der Ich-Erzählerin geht hervor, dass die Mitschüler bei der Identifizierung von Juden keine Schwierigkeiten hatten, denn Juden galten als hinterlistig, gefährlich, bösartig und potthässlich ${ }^{29}$ und waren dadurch leicht $\mathrm{zu}$ erkennen. Die Erzählerin fügt hinzu: „Das hatten wir längst in der Schule und bei den Jungmädchen gelernt, und aus dem Radio auch“30. Die Autorin instrumentalisiert die Kinderfiguren aus der Erzählung der Lehrerin dazu, ihren jungen Lesern einen Einblick in die verbreiteten antijüdischen Vorstellungen $\mathrm{zu}$ geben und führt mit dem folgenden Kommentar des kindlichen Ichs aus, welche Auswirkungen dies in der Vergangenheit auf Kinder und Jugendliche hatte:

Jetzt wurde mir erst richtig klar, warum an manchen Gasthöfen und Cafés ein Schild hing: JUDEN UNERWÜNSCHT! Und warum in den Zeitungen oft so viel Böses über Juden stand. Und warum wir bei den Jungmädchen gelernt hatten, dass wir nicht mit Judenkindern spielen sollen. ${ }^{31}$

Die Kinder waren fest davon überzeugt, dass Juden eine Gefährdung darstellten. Das erwachsene Ich klagt die Lehrerin viele Jahre später an: „Ach Frau Schäfer, was haben sie uns damals angetan!“32. Hat sich die Erzählerin als junges Mädchen noch überzeugen lassen, zeigt sie sich als Erwachsene viele Jahre danach kritisch und urteilssicher. Pausewang kommt auch in dieser Geschichte um die Darstellung und Thematisierung von Tätern und einer gezielten ideologischen Indoktrination nicht

\footnotetext{
26 Pausewang (2004: 46).

27 Pausewang (2004: 47).

28 PAUSEWANG (2004: 48).

29 Pausewang (2004: 47).

30 Pausewang (2004: 47).

31 Pausewang (2004: 48).

32 Pausewang (2004: 49).
} 
herum und lässt in den Schreibprozess der siebten Geschichte zudem autobiographische Elemente einfließen.

In den darauffolgenden Geschichten gegen das Vergessen und Verdrängen konzentriert sich die Autorin auf die verwerflichen Aspekte der Abwehrmechanismen der älteren Generation, auf die Schuldfrage und Schuldvorwürfe vonseiten der Enkelgeneration, die Anlässe dafür schaffen, über die Vergangenheit nachzudenken und die Verantwortung für Verbrechen zu übernehmen. So projiziert Pausewang, für die es kein Verdrängen der Geschichte geben darf, ihre Anliegen einmal mehr auf ihre kindlichen und jugendlichen Protagonisten. Daher verlangt die Enkelfigur aus der Geschichte Das Kopftuch (51-60) nach Aufklärung und versucht zu erfahren, was ihre eigenen Vorfahren in der Vergangenheit getan und unterlassen haben. Sie stößt dabei auf mangelndes Unrechtsbewusstsein und eine ebenso mangelnde Auseinandersetzung der älteren Generation mit der NS-Vergangenheit, woran dann auch jede Kommunikation scheitert. Wenn die Großmutter von der Vergangenheit erzählt, dann so, dass der Anteil und die Schuld der Täter gänzlich aus dem Blick geraten. Franzi ist die einzige in der Familie, die hinterfragt und den gewohnten Ausreden der Großmutter, die zwar als wandelnde Dorfchronik bezeichnet wird, aber nichts von den NS-Verbrechen gehört und gewusst haben will, jede Glaubwürdigkeit entzieht:
„Erzähl mir“, mampft Franzi, „wie es hier in Hornau in der Nazizeit war".
Uffa hält inne und verändert sich plötzlich. Ihr Mund wird zu einem schmalen Strich, ihre Augen verengen sich zu schmalen Schlitzen. Es kommt Franzi vor, als verkrieche sich die alte Frau ganz tief in sich selber. „Wie kommst du denn darauf?“, fragt Uffa finster.
„Wir lernen in der Schule darüber“.
„Diese Lehrer!“, schimpft Uffa. [...]
„Hast du etwa was dagegen“, fragt sie, dass wir die Hornauer Vergan- genheit kennen lernen?“
„Aber doch nicht die!“, knurrt Uffa mit verkniffenem Gesicht.
„Warum nicht die?“, fragt Franzi verblüfft. ,Wir haben ein Recht dar- auf, alle Hornauer Vergangenheiten zu wissen!“ [...]
„Hornau liegt ja so abseits. Hier hat man von dem allem nichts gehört und gesehen“. ${ }^{33}$

Die Enttäuschung über das Verhalten der Großmutter ist groß, die Worte des Geschichtslehrers rütteln das Mädchen aber wieder auf: „Lasst euch nicht abwimmeln. [...] Nur nicht aufgeben [...]. Dranbleiben“"34.

\footnotetext{
33 Pausewang (2004: 53-54).

34 Pausewang (2004: 53-54).
} 
Die vorwurfsvollen Fragen der Enkelin und ihre Hartnäckigkeit bringen die Großmutter dazu, letztendlich nachzugeben. Sie erlaubt damit auch sich selbst, die Vergangenheit zum ersten Mal bewusst in den Blick zu nehmen, Zeichen von Reue und Bedauern über das eigene schuldhafte Verhalten in der NS-Zeit zu zeigen. Die Reaktion der Großmutter, die in Tränen ausbricht und die Hände vor das Gesicht schlägt, macht das deutlich. Ihre Reaktion ist ein Zeichen des Eingeständnisses ihrer persönlichen Schuld. Zum ersten Mal bekennt die Frau ihre Mitschuld an den NS-Verbrechen - sie beaufsichtigte jüdische Gefangene in einer Scheune und lehnte es ab, einer Jüdin zu helfen. Die Verdrängung dieser Ereignisse in der Familie, aber auch die eigene Schuld und Scham verhinderten über viele Jahre hinweg eine offene Aussprache zwischen den Generationen. Der Umgang mit Erinnerungen, die Tendenz zum Nicht-wissen-Wollen, zum Verdrängen und Verharmlosen wird auch in den darauffolgenden Geschichten thematisiert. So informiert sich in der Geschichte Ein Dorf wie jedes andere (105-110) ein junger, neugieriger Tourist über die Vergangenheit des Dorfes Gorbach. Auf die Frage des Touristen, ob es in Gorbach Juden gegeben habe, antwortet ein alter Dorfbewohner:

„Juden? Nein, hier gab es keine“. „Keine einzige Familie?“, [fragt der Tourist]. „Keine. - Oder, warten Sie...ja, Eine einzige. Die Salomons. Die hatten einen Kramladen. Aber der Laden ging Pleite und die Salomons sind weggezogen. Damals war ich noch ein Kind. [...] Jedenfalls gab's hier bei uns keine Judenverfolgungen“. Was für ein beneidenswertes Dorf, denkt der Tourist. Ein Dorf mit gutem Gewissen. Aber der Alte hat sich nur ungenau erinnert, obwohl sein Gedächtnis noch ausgezeichnet arbeitet. Die Familie Salomon lebte bis Ende November 1938 in Gorbach. [...] Und die Alten wissen sehr genau, warum Salomons Laden Pleite ging. Aber sie sprechen nicht darüber. [...] Es darf den schönen Schein Gorbachs ja nicht trüben. Und die mittlere Generation der Gorbacher fragt nicht. Sie weiß zwar, dass da was war. Aber sie weiß auch, dass es unvorteilhaft wäre, in der braunen Vergangenheit ihres Ortes herumzustochern. Damit würde vielleicht allerlei herauskommen, was ihrem Tourismus schaden könnte. Nur das nicht! ${ }^{35}$

Die während der NS-Zeit begangenen Verbrechen wurden in der Öffentlichkeit der Nachkriegszeit und in den NS-belasteten Familien, in denen es eine begründete Angst vor Strafverfolgung gab, ausgeklammert. Pausewang entrollt vor den Augen junger Leser am Beispiel dieser und einiger anderer Geschichten aus der Sammlung gerade dieses Tabui-

35 Pausewang (2004: 106-107). 
sieren, Aussitzen und Verdrängen. Wie anhaltend und vorherrschend diese Einstellung war, wie verbreitet in der Bevölkerung, schildert die Autorin in der Geschichte Ein Dorf wie jedes andere, deren Titel bzw. das nicht benannte Dorf schon darauf hinweist, dass es sich um keinen Einzelfall handelt. Wie dicht das Aufarbeiten und Verdrängen nebeneinander liegen, das führt sie in der Geschichte Besuch aus Amerika (95-104) vor. Im Mittelpunkt der Geschichte steht ein 14-jähriges Mädchen namens Hanna, das über die Ereignisse in ihrer Familie berichtet. Da konsequent aus der Sicht des Mädchens und zunächst über die Gegenwart erzählt wird, ahnen die kindlichen Leser kaum, was in der Familie in der Vergangenheit vorgefallen ist. Der erwachsene Leser erkennt jedoch, dass es in der Familiengeschichte viele Lücken gibt. Was haben die Großeltern und die Urgroßeltern zur Zeit des Nationalsozialismus getan? Wussten sie, was sich damals zugetragen hat? Gab es in ihrem kleinen Dorf in den Bergen Judenverfolgungen? Die Enkelgeneration hat keine eigenen Erinnerungen an das Geschehene, sie ist neugierig und kritisch. Ihr gestiegenes Interesse ist auf die Behandlung des Nationalsozialismus im Geschichtsunterricht sowie auf Fernsehberichte zurückzuführen. Aus dem Unterricht weiß die kindliche Protagonistin, dass die Nationalsozialisten antisemitische, antikommunistische und antidemokratische Positionen vertraten, dass sie für die Judenverfolgung und die Ermordung von Millionen von Menschen verantwortlich sind. Aus Sicht der Großmutter sieht die Vergangenheit heiter aus und die Enkelin ist beruhigt:

Bis in ihr kleines Dorf in den Bergen sei das alles nicht gedrungen. Sie habe erst nach dem Ende der Hitlerzeit von all den schrecklichen Dingen gehört [...]. Ich bin sehr froh darüber, dass Omi und ihre Eltern nichts mit den Nazis zu tun hatten. Denn sie sind ja meine Vorfahren und gehören irgendwie zu mir! ${ }^{36}$

Tatsächlich aber scheut die sonst so erzählfreudige Großmutter Edda vor den Fragen der Enkelin und damit vor einer Auseinandersetzung mit der Vergangenheit zurück. Der zurückhaltenden und offensichtlich schweigenden Großmutter, die in Gesprächen Teile der Familienvergangenheit mit Absicht auslässt oder verharmlost, wird in der Geschichte die redselige Tante aus Amerika gegenübergestellt, die das Mädchen aufklären und zum ersten Mal nach dem Krieg offen über die Täterschaft in der eigenen Familie sprechen möchte. Von der Tante erfährt sie all das, was in der Familie bisher ausgeblendet wurde. Das Mädchen erfährt, dass Großmutters Bruder Dolf nach Hitler benannt wurde, dass der Urgroßvater Ortsgruppenleiter und Onkel Karl Wachmann im KZ

\footnotetext{
36 Pausewang (2004: 97).
} 
Buchenwald war. In der Anschauung der Tante spiegeln sich die Absichten der Autorin wider, die im Nachwort und in verschiedenen Interviews die Notwendigkeit der Tradierung des Wissens und die Bedeutung des Erinnerns betont. Die Absicht der Tante ist das rechtzeitige Aufklären, die Sensibilisierung für wichtige Themen und die Anregung zum Nachdenken über die eigene Vergangenheit.

\section{Synthese}

Gudrun Pausewang gilt als eine der besonders sozialkritischen Schriftstellerinnen Deutschlands. Mit über hundert veröffentlichten Titeln zählt sie heute zu den produktivsten und bekanntesten Kinderbuchautorinnen in Deutschland. Laut einer Statistik des englischsprachigen Wirtschaftsmagazins „Forbes“ gehörte Pausewang außerdem zu den hundert einflussreichsten Frauen Deutschlands. Sie schreibt Geschichten über die Geschichte. Die für diesen Beitrag ausgewählte Geschichtensammlung spiegelt einen Abschnitt deutscher Zeitgeschichte wider und zählt zu den jüngeren Beispielen der Bekenntnisliteratur. Zwar schreibt Pausewang auch für Erwachsene, sie möchte aber an erster Stelle an Kinder und Jugendliche appellieren, unter anderem mit den Geschichten gegen das Vergessen. Am Anfang nahezu jeder Geschichte werden konkrete Angaben darüber gemacht, in welcher Zeit die Handlung spielt und wie alt die Protagonisten sind. Der gemeinsame Nenner der Geschichten ist der Versuch, Erlebtes und subjektiv Erinnertes zu verarbeiten und Erkenntnisse über Kriegskindheiten zu vermitteln. Aufgrund der Analyse entsteht ein Gesamteindruck über die Geschichten aus geschichtsdidaktischer Perspektive. Es ist festzustellen, dass Pausewang gegen das kollektive Vergessen und Schuldgefühle schreibt und zugleich selbst „Bekenntnis“ ablegt. Sie richtet sich dabei ausdrücklich an kindliche Adressaten, die weder am Ausgrenzungs- noch am Vernichtungsprozess beteiligt sind (mit Ausnahme der Geschichte Treibjagd, in der ein 15-Jähriger auf Anstachelung seines Onkels einen russischen Kriegsgefangenen erschießt). Sie möchte aufklären und das Bewusstsein junger Leser für die Vergangenheit sensibilisieren. Jede einzelne Geschichte transportiert diese Intention und offenbart somit die politische und gesellschaftliche Positionierung der Autorin. Als Angehörige der Zeitzeugengeneration wendet sie sich an die jüngste Generation, die über keine eigenen Erinnerungen an die NS-Zeit verfügt, und leistet insbesondere dort, wo sie den Nationalsozialismus und Holocaust thematisiert, einen wichtigen Beitrag zur Herausbildung und Vermittlung von historischem Wissen und moralischen Werten. 
In den lose miteinander verbundenen Geschichten stehen kindliche oder jugendliche Helden im Mittelpunkt der Handlung, die sich durch ihre Kürze und Prägnanz auszeichnet. Die kindlichen und jugendlichen Protagonisten sind zwischen zehn und siebzehn Jahren alt und ideologisch durch eine aggressive Propaganda verblendet. Die Mädchen- und Jungenfiguren sind in den zwanzig Geschichten ungleichmäßig verteilt. Bei einem einfachen Nachzählen der Figuren in den Geschichten ergibt sich: in insgesamt vierzehn Geschichten kommen Mädchenfiguren vor, Jungenfiguren dagegen nur in sechs Geschichten. Erwachsene werden in der Geschichtensammlung als sich Bereichernde (Er war noch warm), als in listiger Vorausschau Handelnde (Der Persilschein), als die NS-Ideologie Mittragende (Das Gespräch u.a.) und als Schweigende (Das Kopftuch, Ein Dorf wie jedes andere, Besuch aus Amerika) dargestellt. Die Täteranteile werden in den Geschichten Das Kopftuch, Ein Dorf wie jedes andere und Besuch aus Amerika entweder ausgeblendet oder von der älteren Generation umgedeutet.

Alle zwanzig Geschichten, insbesondere aber die Geschichten Das Mutterkreuz, Ein Novembermorgen oder etwa die Geschichte Märchenstunde, machen auf nationalsozialistische Erziehungsmaßnahmen in der Schule und in Jugendorganisationen aufmerksam. Sie schildern aber auch das Durchgreifen der ideologischen Vorstellungen des NS-Regimes außerhalb des Unterrichts, wie etwa in den Geschichten Es war noch warm, Der Persilschein oder in der Geschichte Treibjagd. Pausewang nimmt im Nachwort zu der gezielten ideologischen Indoktrination Stellung:

Wir jungen Menschen bekamen damals diese NS-Ideologie über die Schule, die Medien, die Jugendorganisationen eingetrichtert. Sehr viele von uns auch über die Eltern, die ja unsere Vorbilder sein wollten. Natürlich glaubten wir denen, die uns indoktrinierten. Wir hatten ja nie gelernt kritisch zu überdenken, was man uns beibrachte. ${ }^{37}$

Genau so stellt die Autorin ihre kindlichen und jugendlichen Protagonisten dar, leicht beeinflussbar und grundsätzlich nicht in der Lage, das Handeln von Erwachsenen zu hinterfragen. Ein weiteres Merkmal kind- und jugendgemäßer Texte ist die Wahl einer einfachen Sprache und auch der paratextuelle Bereich weist bei Pausewang einen kindund jugendgemäßen Charakter auf. Die Kritik, dass ihre Texte Kindern nicht zumutbar seien, kann Pausewang nicht nachvollziehen und erklärt: „Auch politische, ökologische oder historische Themen verdauen

\footnotetext{
37 Pausewang (2004: 153).
} 
sie ohne Schwierigkeiten, wenn man sie ihnen altersgerecht anrichtet“"38. Sie führt mit ihren Texten vor, wie die NS-Vergangenheit literarisch angemessen festgehalten werden kann, ohne die jungen Leser zu überfordern oder gar zu traumatisieren. Andere Kinder- und Jugendbuchautoren teilen Pausewangs Ansicht. So meint etwa Torsten Körner am Ende seines Jugendbuches Die Geschichte des Dritten Reiches (2000):

Nur wenn man sich mit der dunklen Geschichte des Dritten Reichs beschäftigt und aus ihr lernt, kann man verhindern, dass sich ähnliche finstere Kapitel in der Zukunft wiederholen. [...] Neue Wege und bessere Möglichkeiten tun sich nur dann auf, wenn man sich die Irrwege und schrecklichen Beispiele der Geschichte vor Augen führt. Wie wir unsere Zukunft gestalten, müssen wir selbst bestimmen. Das ist unsere Verantwortung. Wie sie nicht aussehen soll, hat uns die Geschichte des Dritten Reiches gezeigt. ${ }^{39}$

Die Autoren leisten mit ihren geschichtserzählenden Kinder- und Jugendbüchern einen bedeutenden Beitrag zur politischen Bildung der Kinder und Jugendlichen. Zum Schluss sei angemerkt, dass Pausewangs Geschichtensammlung sicherlich auch als Appell an die junge Leserschaft, über die eigene Familiengeschichte nachzudenken, gedeutet werden kann.

\section{Literatur}

Aden-Grossmann Wilma (2000): Die Epoche des Nationalsozialismus in der Kinder- und Jugendliteratur. In: Nolz Bernhard / Popp Wolfgang (Hg.): Erinnerungsarbeit. Grundlage einer Kultur des Friedens. Münster: LIT Verlag: 285-297.

ANONYm (2013): Gudrun Pausewang wird 85. In: URL: http://www.focus.de/kul tur/buecher/literatur-gudrun-pausewang-wird-85_aid_930122.html [Zugriff am 2.10.2017]

Assmann Aleida (2006): Generationsidentitäten und Vorurteilsstrukturen in der neuen deutschen Erinnerungsliteratur. Wien: Picus Verlag.

Assmann Aleida (2011): Was bedeutet eigentlich Erinnerung? - Aleida Assmann im Gespräch. In: https://www.goethe.de/ins/br/de/kul/fok/cul/20809570. html [Zugriff am 19.9.2018].

DAHRENDORF Malte (1997): Das zeitgeschichtliche Kinder- und Jugendbuch zum Thema Faschismus/Nationalsozialismus: Überlegungen zum gesellschaftlichen Stellenwert, zur Eigenart und Didaktik. In: Rank Bernhard / Rosebrock Cornelia (Hg.): Kinderliteratur, literarische Sozialisation und Schule. Schrif-

\footnotetext{
38 Pausewang (2004: 32-33).

39 KÖRNER (2000: 153).
} 
tenreihe der Pädagogischen Hochschule Heidelberg. Weinheim: Deutscher Studienverlag: 201-226.

Diner Dan (1987): Negative Symbiose. Deutsche und Juden nach Auschwitz. In: Diner Dan (Hg.): Ist der Nationalsozialismus Geschichte? Zur Historisierung und Historikerstreit. Frankfurt am Main: Verlag Fischer: 188-189.

Ewers Hans-Heino / Gremmel Caroline (2008): Zeitgeschichte, Familiengeschichte und Generationenwechsel. Deutsche zeitgeschichtliche Jugendliteratur der 1990er und 2000er Jahre im erinnerungskulturellen Kontext. In: GLASENAPP Gabriele von / EwERs Hans-Heino (Hg.): Kriegs- und Nachkriegskindheiten. Studien zur literarischen Erinnerungskultur für junge Leser. Frankfurt am Main: Peter Lang: 27-50.

Ewers Hans-Heino (2012a): Kinder- und Jugendliteratur. In: http://www.kinder undjugendmedien.de/index.php/begriffe-und-termini/411-kinder-und-jugend literatur [Zugriff am 20.9.2017].

Ewers Hans-Heino (2012b): Literatur für Kinder und Jugendliche. Paderborn: UTB.

JeLeč Marijana (2016): Formen der Vergangenheitsbewältigung in ausgewählten zeitgenössischen österreichischen Generationenromanen. In: LovRIć Goran / Jeleč Marijana (Hrsg.): Familie und Identität in der Gegenwartsliteratur. Frankfurt am Main: Peter Lang: 147-162.

Kelm Heike (2006): Autobiographische Spuren im Narrativ ausgewählter deutscher Kinder- und Jugendbuchautoren der Kriegs- und Nachkriegsgeneration. Eine qualitative Studie. Dissertation. Münster.

KöRnER Torsten (2000): Die Geschichte des Dritten Reiches. Frankfurt am Main: Campus Verlag.

L'Aввате Valentina (2014): Gudrun Pausewangs politische Werke: politische Kinder- und Jugendliteratur im Medienzeitalter. Hamburg: Diplomica Verlag.

Lenz Siegfried (1992): Über das Gedächtnis. In: Lenz Siegfried: Über das Gedächtnis. Reden und Aufsätze. Hamburg: Deutscher Taschenbuch Verlag.

NösTlinger Christine (1986/87): Ist Kinderliteratur Literatur? In: Oetinger Lesebuch 23: 158-164.

Pausewang Gudrun (1999): Hallo, Vetter Quijote: Rede in der Johann Wolfgang Goethe-Universität am 02. Juli 1999. In: Freundeskreis des Instituts für Jugendbuchforschung der Johann Wolfgang Goethe-Universität (Hg.). Frankfurt am Main.

Pausewang Gudrun (2004): Ich war dabei. Geschichten gegen das Vergessen. Frankfurt am Main: Fischer Kinder- und Jugendtaschenbuch.

Ramm Daniel (2015): Interview mit Gudrun Pausewang. In: https://www.stern. de/nido/interview-gudrun-pausewang-7155204.html [Zugriff am 19.9.2018].

Runge Gabriele (1991): Über Gudrun Pausewang. Ravensburg: Ravensburger Buchverlag.

SteINLEIN Rüdiger (1996): Deutschsprachige Kinder- und Jugendliteratur zum Thema Nationalsozialismus. In: Deutschunterricht 49: 87-96.

WILD Reiner (2002): Geschichte der deutschen Kinder- und Jugendliteratur. Stuttgart-Weimar: Verlag J. B. Metzler. 


\begin{abstract}
"Die Wahrheit ist den Kindern zumutbar" - Schuld und Bekenntnis in Gudrun Pausewangs geschichtserzählendem Kinder- und Jugendbuch Ich war dabei. Geschichten gegen das Vergessen
\end{abstract}

Zusammenfassung: Der Beitrag widmet sich Gudrun Pausewangs geschichtserzählendem Kinder- und Jugendbuch Ich war dabei. Geschichten gegen das Vergessen (2004) und setzt sich mit der Inszenierung von Geschichte sowie mit der Erkenntnis und Bekenntnis von Schuld in der zeitgenössischen Kinder- und Jugendliteratur auseinander. Der Beitrag untersucht, auf welche Art und Weise und mit welcher Absicht die Themenkomplexe Nationalsozialismus und Holocaust in die deutschsprachige Kinder- und Jugendliteratur von Pausewang aufgenommen werden. Im Verlauf der Untersuchung wird deutlich, dass auch die Kinder- und Jugendliteratur einen geeigneten Ort für die Auseinandersetzung mit dem Nationalsozialismus bietet und einen Beitrag zur politischen Bildung der Kinder und Jugendlichen leistet. Die Geschichten führen vor, wie Kinder und Jugendliche mit der Durchsetzung der nationalsozialistischen Ideologie umgehen und zeigen traditionelle Muster der Verdrängung auf. Pausewang weist mit ihren Geschichten auf die individuelle und kollektive Bedeutung der Vergangenheitsaufarbeitung in Kinderbüchern hin.

Schlagwörter: Kinder- und Jugendliteratur, Nationalsozialismus, Indoktrination, Schuld, Bekenntnis

"Dzieci poradza sobie z prawda" - wina i przyznanie się
do winy w powieści historycznej dla dzieci i młodzieży Ich
war dabei. Geschichten gegen das Vergessen Gudrun Pausewang

Streszczenie: Autorka artykułu analizuje książkę historyczną dla dzieci i młodzieży Ich war dabei. Geschichten gegen das Vergessen (2004) Gudrun Pausewang i koncentruje się na problemie przedstawiania historii, rozpoznawania winy i przyznawania się do niej w kontekście współczesnej literatury dla dzieci i młodzieży. Bada, w jaki sposób i w jakim celu wprowadzana jest do literatury dla dzieci i młodzieży tematyka narodowego socjalizmu i holocaustu. Wykazuje, że również literatura dla dzieci i młodzieży umożliwia rozliczenie z narodowym socjalizmem i przyczynia się do politycznego kształcenia dzieci i młodzieży. Analizowane opowiadania problematyzują tradycyjne wzorce wypierania winy i pokazują, jak dzieci i młodzież podchodzą do propagowania ideologii nazistowskiej. Pausewang wskazuje swoimi opowiadaniami na indywidualne i zbiorowe znaczenie rozliczania się z przeszłością w książkach dla dzieci.

Słowa kluczowe: literatura dla dzieci i młodzieży, narodowy socjalizm, indoktrynacja, wina, przyznanie się do winy 
'Children will cope with the truth' - guilt and admission to guilt in the historical novel for children and teenagers Ich war dabei. Geschichten gegen das Vergessen by Gudrun Pausewang

\begin{abstract}
The author of the article analyses the historical book for children and teenagers Ich war dabei. Geschichten gegen das Vergessen (2004) by Gudrun Pausewang and focuses on the problem of presenting history, recognizing and admitting guilt in the context of contemporary literature for children and teenagers. The author examines in what way and for what purpose the topic of national socialism and holocaust is introduced into the literature. She demonstrates that literature for children and teenagers can also be a genre that makes it possible to deal with national socialism and can contribute to the political education of children and youngsters. The stories subjected to analysis address traditional patterns of guilt suppression and show how children and teenagers approach the propagation of Nazi ideology. Pausewang points with her stories to the individual and collective importance of settling accounts with the past in books for children.
\end{abstract}

Keywords: literature for children and teenagers, national socialism, indoctrination, admission to guilt

Marijana Jeleč ist Dozentin an der Abteilung für Germanistik der Universität Zadar und Gastdozentin an der Pädagogischen Fakultät der Universität Bihać. Sie studierte Germanistik in Zadar, promovierte 2014 mit einer Analyse von österreichischen Familienromanen nach 2000. Zu ihren Forschungsschwerpunkten gehören: Familien- und Generationenromane, Kinder- und Jugendliteratur, Narrative der Flucht, literarische Inszenierung von Geschichte, Erinnerungs-, Verdrängungs- und transgenerationellen Übertragungsprozessen. Letzte Publikationen: Obitelj $i$ povijest u suvremenom austrijskom generacijskom romanu (dt. Familie und Geschichte im zeitgenössischen österreichischen Generationenroman). Zagreb 2015; (Hg.) Familie und Identität in der Gegenwartsliteratur. Frankfurt am Main 2016; Routen der Flucht und hybride Identitäten in Abbas Khiders autobiographisch inspirierten Exilromanen „Der falsche Inder“ (2008) und „Ohrfeige“ (2016). Riga 2018.

Marijana Jeleč jest docentem w Zakładzie Germanistyki na Uniwersytecie w Zadarze, wykłada gościnnie na Wydziale Pedagogicznym Uniwersytetu w Bihaciu. Studiowała germanistykę w Zadarze, doktoryzowała się w 2014 roku na podstawie analizy austriackich sag rodzinnych publikowanych po roku 2000. Zainteresowania naukowe: sagi rodzinne, literatura dla dzieci i młodzieży, motyw ucieczki, literackie przedstawianie historii, problematyka pamięci i wypierania w komunikacji międzypokoleniowej. Najnowsze publikacje: Obitelj i povijest u suvremenom austrijskom generacijskom romanu (niem. Familie und Geschichte im zeitgenössischen österreichischen Generationenroman). Zagreb 2015; (Hg.) Familie und Identität in der Gegenwartsliteratur. Frankfurt am Main 2016; Routen der Flucht und hybride Identitäten in Abbas Khiders autobiographisch inspirierten Exilromanen „Der falsche Inder“ (2008) und „Ohrfeige“ (2016). Riga 2018. 